\title{
Sonification of weather data as a non-human-centric artistic
}

\section{approach [version 1; peer review: 1 not approved]}

\author{
Yi Kee Ng(D), Kok Yoong Lim(D) \\ Faculty of Creative Multimedia, Multimedia University, Cyberjaya, Selangor, 63100, Malaysia
}

V1 First published: 26 Jan 2022, 11:96

https://doi.org/10.12688/f1000research.73543.1

Latest published: 26 Jan 2022, 11:96

https://doi.org/10.12688/f1000research.73543.1

\begin{abstract}
Background - In the mid-20th century, the emergence of sound studies demonstrated a shift of research interest in sonic practitioners. This field gains its prevalence by expanding the boundaries of prevailing conception through proposing alternative creative approaches in sound art practices.

Methods - Two methods were presented - listening and sounding to promote creative sound making. The first method, listening involves soundwalking and recording sound from the external environments. These recordings were then re-evaluated and post-processed in audio editing software. The second method, sounding involves the creation of a weather data sonification system in Pure Data environment, in which the perceptual experience from the first method was taken into consideration.
\end{abstract}

Result - First method enables the genesis of creative idiosyncrasies, such as preferences and ideas through the sonic perception of environmental events. In this process, noise and weather were identified as environmental components that share similar sensible qualities. Thus, noise is a prevalent medium that inspires the creation of sound generators in the sonification system presented in this paper. The sonic output of data sonification reveals an analogical connection between weather data and sonic parameters, in which changes in data values result in changes in acoustic properties. These outputs deliver different sensibilities based on their data parameters; sonification of temperature data might suggest an alarming effect to the listener.

Conclusion - The proposed methods were intricately linked, suggesting environmental events to be perceived and realized through a non-scientific perspective. By highlighting aesthetic possibilities of environmental components, this paper presents an alternative perspective in contrast with the human-centric worldview through the creation of sonic works.

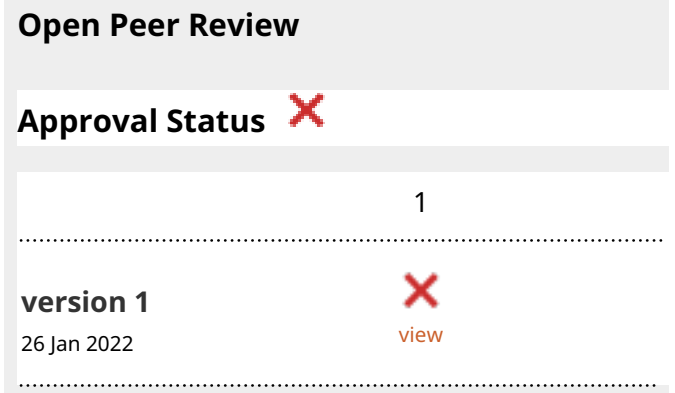

1. Tin Oberman (D), University College London, London, UK

Any reports and responses or comments on the article can be found at the end of the article. 


\section{Keywords}

Non-human-centric perspectives, sonification, sound design, sonic parameters, weather data

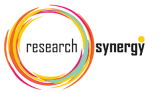

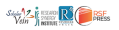

This article is included in the Research Synergy

Foundation gateway.

Corresponding author: Kok Yoong Lim (kylim@mmu.edu.my)

Author roles: Ng YK: Conceptualization, Investigation, Methodology, Resources, Software, Visualization, Writing - Original Draft Preparation; Lim KY: Funding Acquisition, Project Administration, Supervision, Validation, Writing - Review \& Editing

Competing interests: No competing interests were disclosed.

Grant information: The author(s) declared that no grants were involved in supporting this work.

Copyright: @ $2022 \mathrm{Ng}$ YK and Lim KY. This is an open access article distributed under the terms of the Creative Commons Attribution License, which permits unrestricted use, distribution, and reproduction in any medium, provided the original work is properly cited.

How to cite this article: $\mathrm{Ng}$ YK and Lim KY. Sonification of weather data as a non-human-centric artistic approach [version 1; peer review: 1 not approved] F1000Research 2022, 11:96 https://doi.org/10.12688/f1000research.73543.1

First published: 26 Jan 2022, 11:96 https://doi.org/10.12688/f1000research.73543.1 


\section{Introduction}

In the 21 st century, sound studies research ${ }^{1}$ prevailed as an academic field to investigate and reflect on the continuous establishment of strategies, culture, and aesthetics, amid the emergence of new sonic practices. The research was categorized into different tropes which focused on sonic perception, sonic sites and soundscapes, sonic reproduction, artists and collectives, and sonic aesthetics.

Therefore, the strategies and creative possibilities of sonic methods under the category of soundscape were investigated. In other literature, the term soundscape was defined as 'events heard, not objects seen', whereas in this research it was defined as a discipline that examines the effects of the acoustic environment on the creatures and entities living within it. This then contributes to the establishment of various noise abatement and musical practices as methods of advocating and appreciating the beauty of environmental soundscape. ${ }^{2}$ Although soundscape research started as a musical endeavour for appreciation of environmental sound, which proclaimed the world as a 'macrocosmic musical composition', it only focused on collecting and examining 'non-polluted' sound through field recordings. Thus, this contradiction offers the possibilities of sonic practitioners to extend and practicalize its original concepts into other creative practices.

Despite this, soundscape research encouraged sonic practitioners and non-practitioners to engage with the environment through listening. Instead of focusing on sound from the natural environment, works from avant-garde practitioners explore the creative possibilities of sound indiscriminately from different environments. In Sonic Meditations, ${ }^{3}$ the composer Oliveros encouraged a series of sonic experimental activities such as listening and recording environmental sounds, journaling listening experiences and producing sound works as part of deep listening practice. On the contrary, Feld's acoustemological approach derived from a non-human perspective- to acknowledge relation shared with numerous human and non-human actants, and ultimately the construct of the world through 'sonic ways of knowing'.

On a similar notion, this paper will engage and discuss human and non-human relations through sonic means. We propose two methods - listening and sounding. First, listening and soundwalking were conducted in order to observe and examine the overlap of sonic components emitted from human and non-human influences in the physical environment. In hopes to better understand other non-sonic events of the environment, weather data was collected through a virtual open-source platform. Second, sounding involves analysing, post-processing and re-evaluating sound samples that were recorded per $s e$. The findings of the analysis enable relation to be drawn between sonic properties and quality, which then guide the process of data sonification.

\section{Methods}

Method 1: Listening and data collection

Listening in the context of this paper draws closely on the methods that were promoted in previous deep listening practices. ${ }^{6}$ This involved observing and recording sonic events simultaneously when navigating through the sonic landscapes of different environments. In short, this method of perceiving defined soundwalking practice.

The process of soundwalking first involved observing and listening attentively to a different sound and sonic events that took place in the surrounding environment. This process was facilitated by using a digital audio recorder (Zoom H1n) which enables sound with different properties to be heard and detected as audio signal input. In other words, this facilitation intensified the listening experience, in which sound with lower amplitudes and high frequencies that were less audible, such as forest ambience, bird songs were amplified and heard through the device's integrated stereo microphones. Thus, based on the properties of sound, the gain of signal inputs was monitored and adjusted accordingly.

Then, soundwalking proceeded with identifying and recording environmental sound with desirable sensible qualities, specifically surrounding ambience that was often perceived as 'noise' or 'insignificant sound'. Based on the understanding that sound possessed distinctive qualities in different spatiotemporal settings, soundwalking was conducted in both natural and urban environments. Decisions such as moving towards or away from the sound source were made on-site based on the changing qualities of the environment. Each recording was recorded in 5-7 minutes, thus durationally these recordings can be perceived as sonic events that documents the multiplicities of sonic components emitted from non-human and human producers.

Soundwalking enables environmental events to be perceived primarily through sonic means, however, these events can also be perceived through other technological means. Hence, we identify weather as events that share similar sensible qualities with ambience recordings, that of being 'unpredictable' and 'indeterminate'. In order to examine the changing process of weather events on a specific timeline, weather data of the soundwalking site was collected through a virtual open-source platform known as Open Weather Map (OWM) that integrates several data sources such as numerical weather prediction (NWP), weather stations and satellite data. ${ }^{7}$ The data was retrieved in numerical format through 


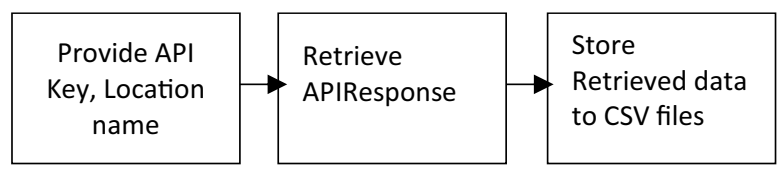

Figure 1. Integrating OWM API in Python environment.

Application Programming Interface (API) calls and stored externally in comma-separated values (CSV) files, as shown in Figure 1. Hence, weather data collection can be seen as an extension of soundwalking - a method of environmental engagement.

\section{Method 2: Sounding, data analysis and sonification}

Recordings of the sonic events were categorized based on the details of recording sites, sonic components and sources. For example, sound recordings of forests were categorized as the natural soundscape that consists of bird song, wind noise and hums, whereas sound recordings of cityscape were categorized as the urban soundscape that consists of noises of transportation and traffic as some of the major sonic components. These details were documented to ensure future navigation.

In order to narrow down the variables of recordings, this paper only focuses on the sound that shares similar sensible qualities with the environment. The sound emitted from other sources such as birds, people and transportation were not sampled or used for analysis. Hence, the sound of the surrounding ambience such as the noise of wind and other geophysical influences were sampled out from the recordings into a shorter duration. These sampled sounds were most often perceived as 'background noise' or rumbles, in which the sources of emission were arbitrary or unrecognizable. To examine the sensible qualities of background ambience, the acoustic properties of different samples were compared through audio analysis tools, namely a frequency and amplitude follower.

We selected samples from both urban and natural environments; the comparison is better visualized in the form of spectrograms. The values of amplitude $(\mathrm{dB})$ and frequency $(\mathrm{Hz})$ were annotated on each spectrogram. The analysis was done with Sonic Visualizer. The figures below depict the result of acoustic analysis across a specific duration: Figure 2 shows a higher fluctuation of both amplitude and frequency as compared to Figure 3. The comparison of acoustic properties was summarized in Table 1.

The difference in acoustic properties result in the difference of sensible qualities: samples of cityscape could be perceived as loud, chaotic and noisy, whereas samples of forest could be perceived as soothing, calming and unobtrusive. These
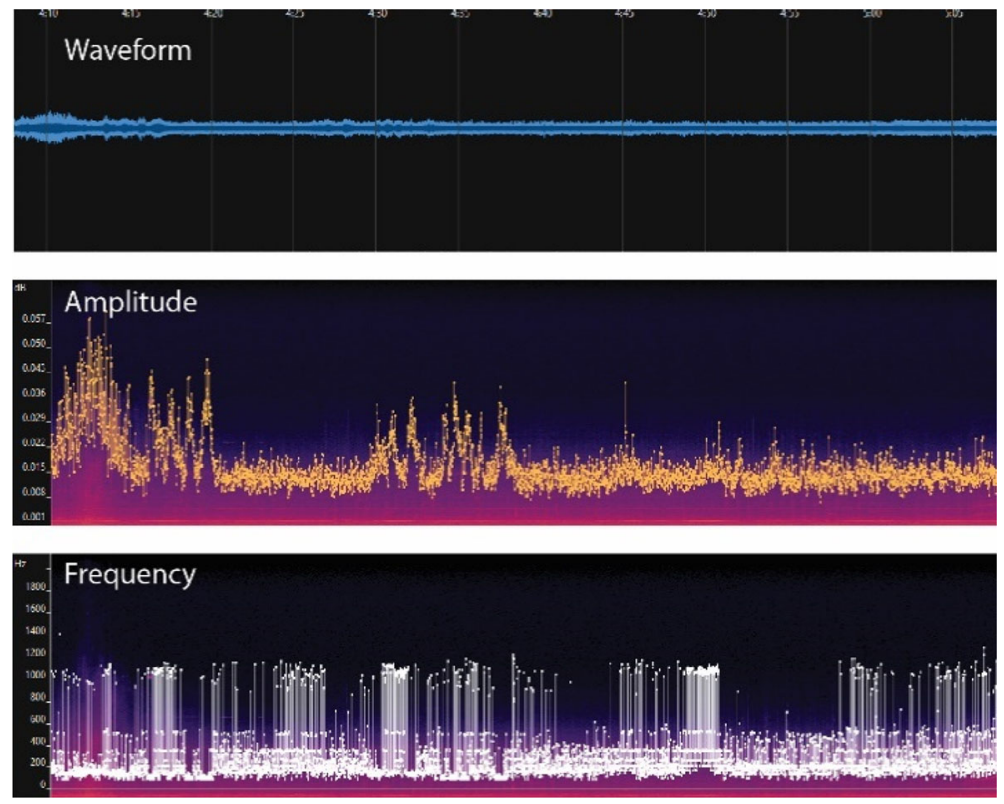

Figure 2. Spectrogram of waveforms, amplitude and frequency of sound recorded in a cityscape. 

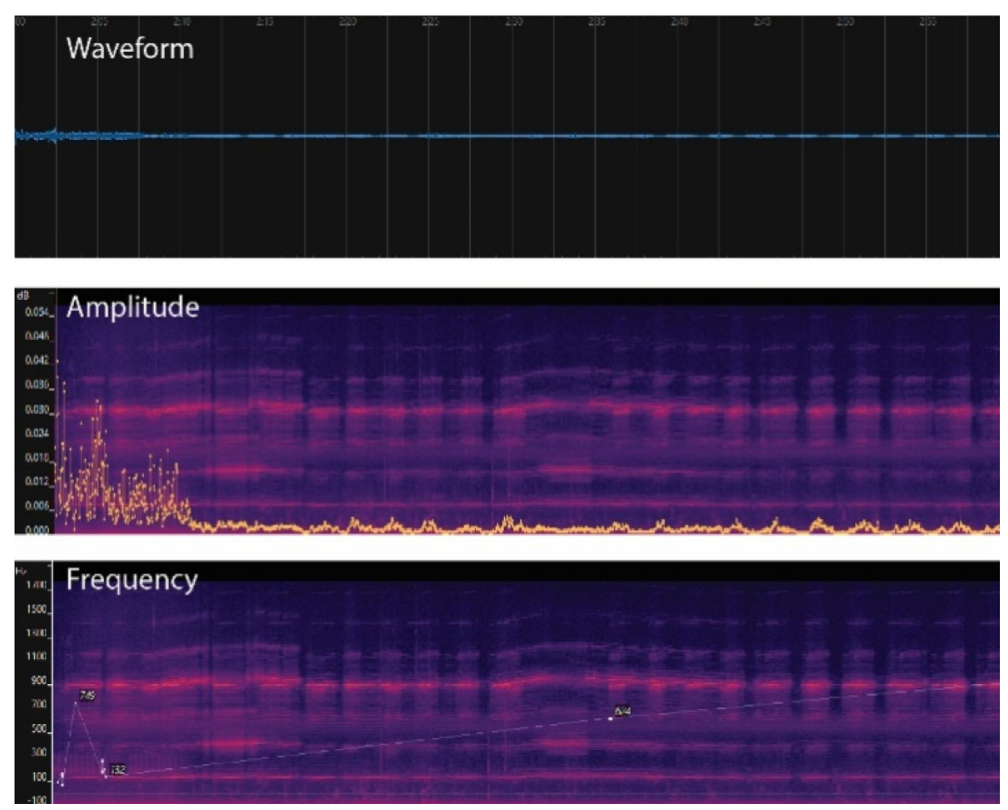

Figure 3. Spectrogram of waveforms, amplitude and frequency of sound recorded in a forest.

Table 1. Comparison of acoustic properties.

\begin{tabular}{|l|l|l|l|}
\hline Recording & Sampled duration & Amplitude range (dB) & Frequency range (Hz) \\
\hline Cityscape (Figure_) & 1 Minute & $0.006637-0.060209$ & $93.75-1607.03$ \\
\hline Forest (Figure_) & 1 Minute & $0.000375-0.061951$ & $70.1189-748.849$ \\
\hline
\end{tabular}

identified qualities enable the genesis of idiosyncratic preferences, knowledge and sensitivity on the sensibilities of noise. Thus, it indirectly inspires and influences the decision-making process of creating sound generators in the sonification process.

Sound generators were created in Pure Data (PD) - an open -source visual programming environment to sonify different parameters of weather data that were collected previously. The sonification process translates numerical data into sonic outputs - in which sonic parameters of generators were modified by incoming data parameters. ${ }^{8}$ Each data point maps to the frequencies and amplitudes as shown in Table 2. Consequently, this mapping allows the changes of data points to be revealed and perceived across time.

To distinguish weather data parameters from one another, the parameters were assigned to three different sound generators which were synthesized to deliver different sensible qualities. In order to represent the distinctive changes of temperature data, a sawtooth wave oscillator with an amplitude envelope of short release time was used to deliver a sharper sonic quality. In contrast, to represent the fluid and irregular physical quality of clouds, a sine wave oscillator and white noise generator were used; enhanced with slight reverberation to ensure a smoother sounding quality. Lastly, rain volume was represented with white noise generators by structures of high-pass and low pass filters to mimic rainy ambience. Figure 4 shows examples of sound generators in the sonification system.

\section{Table 2. Data transmission of the sonification system.}

\begin{tabular}{|l|l|l|l|}
\hline Sound generators & Main generator & Input data parameters & Modified sonic parameters \\
\hline 1 & Sawtooth wave oscillator & Temperature & Frequencies \\
\hline 2 & Sine wave oscillator & Cloud coverage & Frequencies, amplitude of noise \\
\hline 3 & White noise oscillator & Rain volume & Amplitude \\
\hline
\end{tabular}




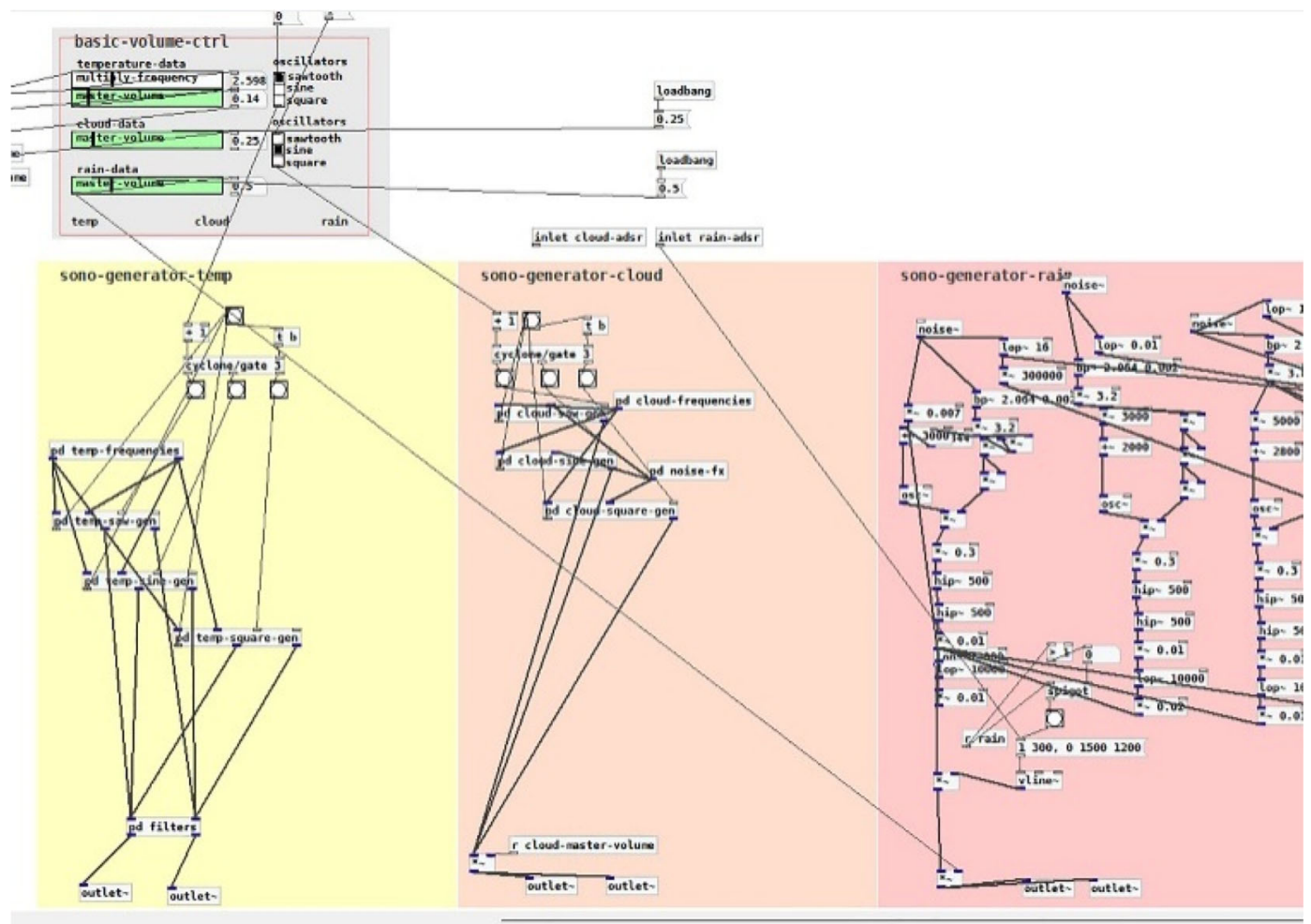

Figure 4. Sound-generators for temperature (left) and cloud coverage (right) in PD.

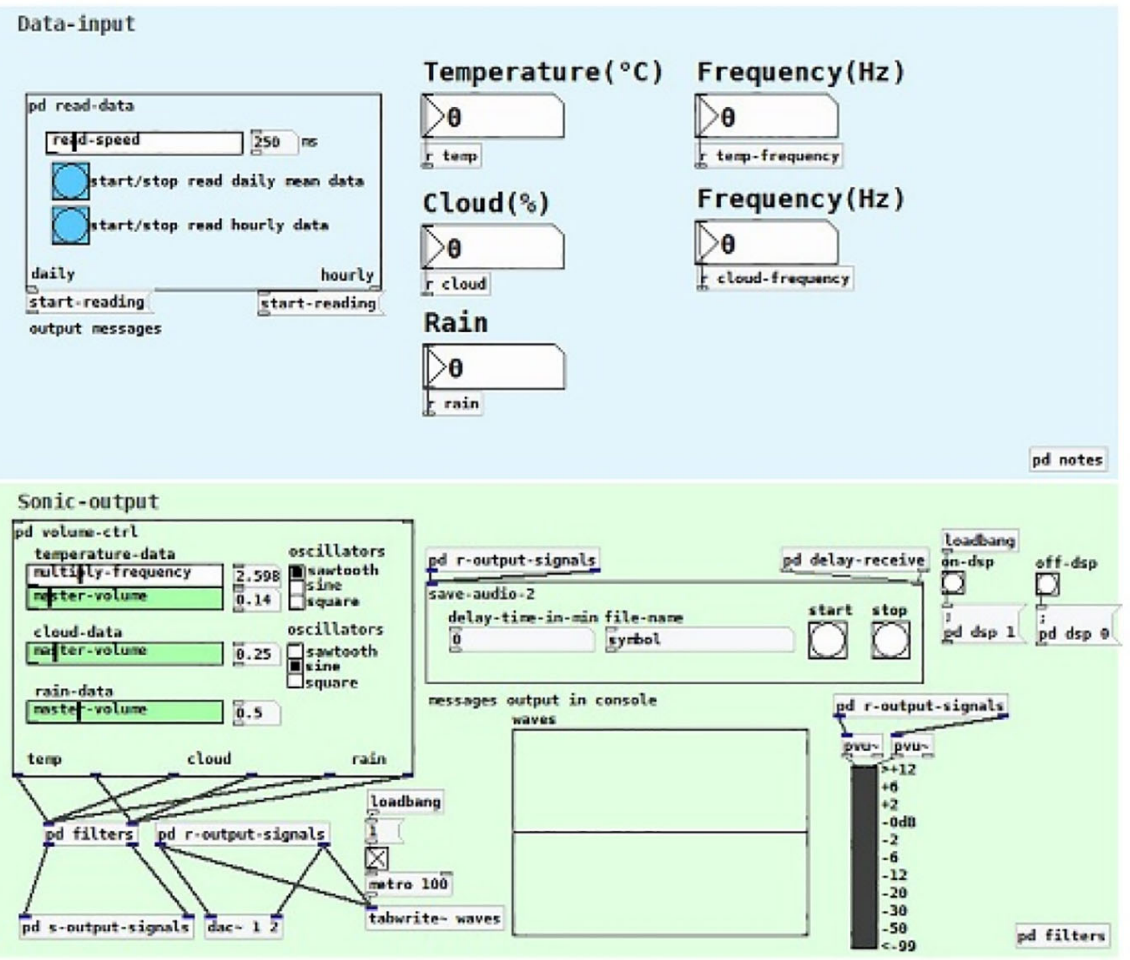

Figure 5. Graphic User Interface (GUI) for controlling parameters of data input and output. 
To afford control in the process of sonification, the system was designed with a few interactive parameters. The front-end of the system was organized into two parts as shown in Figure 5, the top part display control parameters for input weather data, which consist of two options of data sets and controllable data reading intervals (milliseconds), whereas the bottom part display volume control $(\mathrm{dB})$ for sonic output. In consequence, the sonic output varies each time when the control parameters were modified.

For a desirable result, the control parameters were set constant. Daily data was chosen from the dataset and its reading interval was set to 350 milliseconds, which means each data point was read and translated into sound between the given time intervals. Weather data parameters, namely temperature, cloud coverage and rain volume were sonified and exported separately into three (.wav) files with a duration of 3 minutes. Each sonification output was later visualized in spectrogram to draw a relation between weather data and sonic parameters.

\section{Results}

Practicalizing sonic methods to perceive and re-enact sensibilities of environmental events

In this paper, we propose sonic methods as practical ways of perceiving and re-enacting sensibilities of environmental events. This proposition presupposes events derived from everyday reality are possible catalysts for artistic creation. Through practicalizing these methods, a relation was drawn between environmental components and intricacies that were perceived, both through sonic and non-sonic means. In the first method, noise, ambience, and weather were identified as specific components that share similar sensible qualities, that of being unpredictable and random in its materialities. That being the case, the noise was highlighted as a prevalent sonic medium that inspires the creation of sound generators which were later used to represent the sensibilities of weather data through sonification.

Environmental perception enables the formation of idiosyncratic experience, ideas, preferences, and affords ways of realizing and re-enacting sensibilities. In the second method, sonification was seen as a method in realizing and re-enacting sonic experiences. This method considers how weather events were experienced, for example, how temperature changes seemingly deliver an alarming effect, or how moving clouds might suggest a sense of wonder. Thus, each data parameter was mapped onto different sound generators to deliver distinctive sensibilities. Sonic output of temperature data was heard as distinctive changes in frequencies; cloud data was heard as subtle changes in frequencies, random changes in amplitude of noise; and rain was heard as ambience based on its occurrences. The outputs suggest an analogical connection between weather data parameters and sonic parameters, this connection is visualized in spectrograms, as shown in Figures 6, 7 and 8.

The spectrograms suggested the most distinctive changes in the acoustic properties of temperature sonification as compared to others. This method of outputting sonification process enables weather data to be represented as distinctive
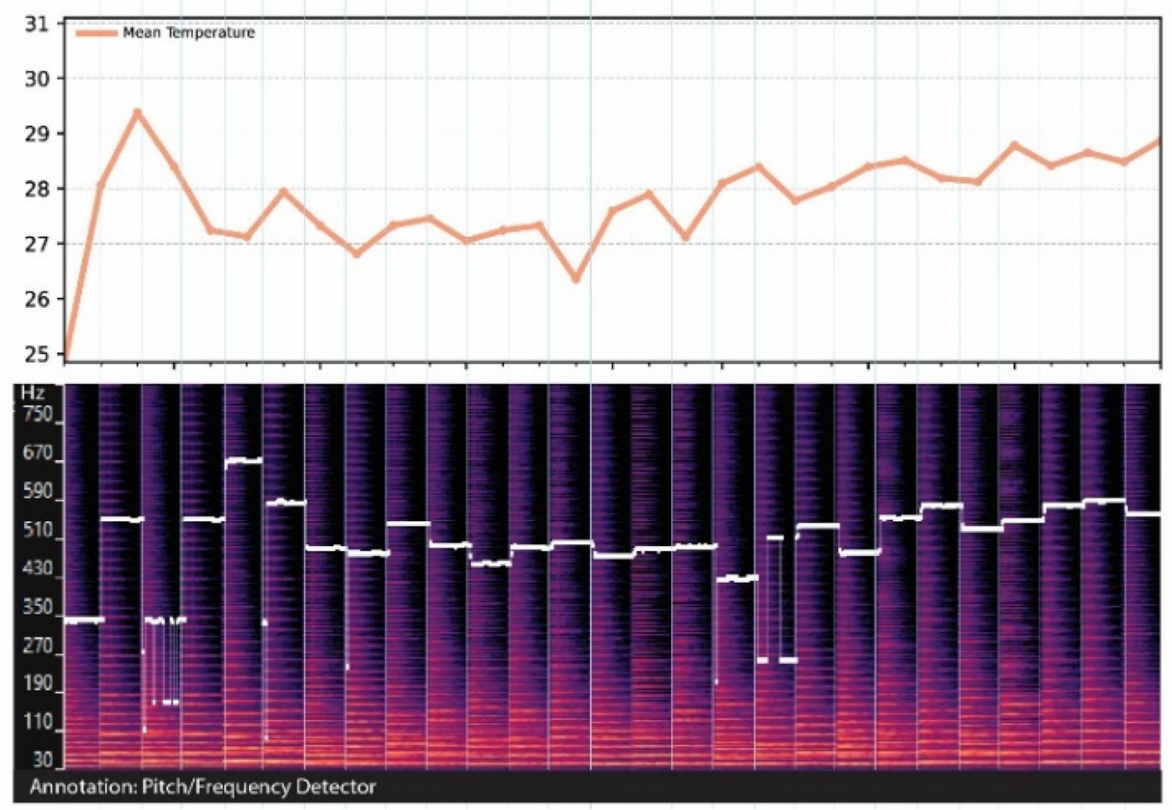

Figure 6. Comparison of changes in temperature data and changes in frequencies. 


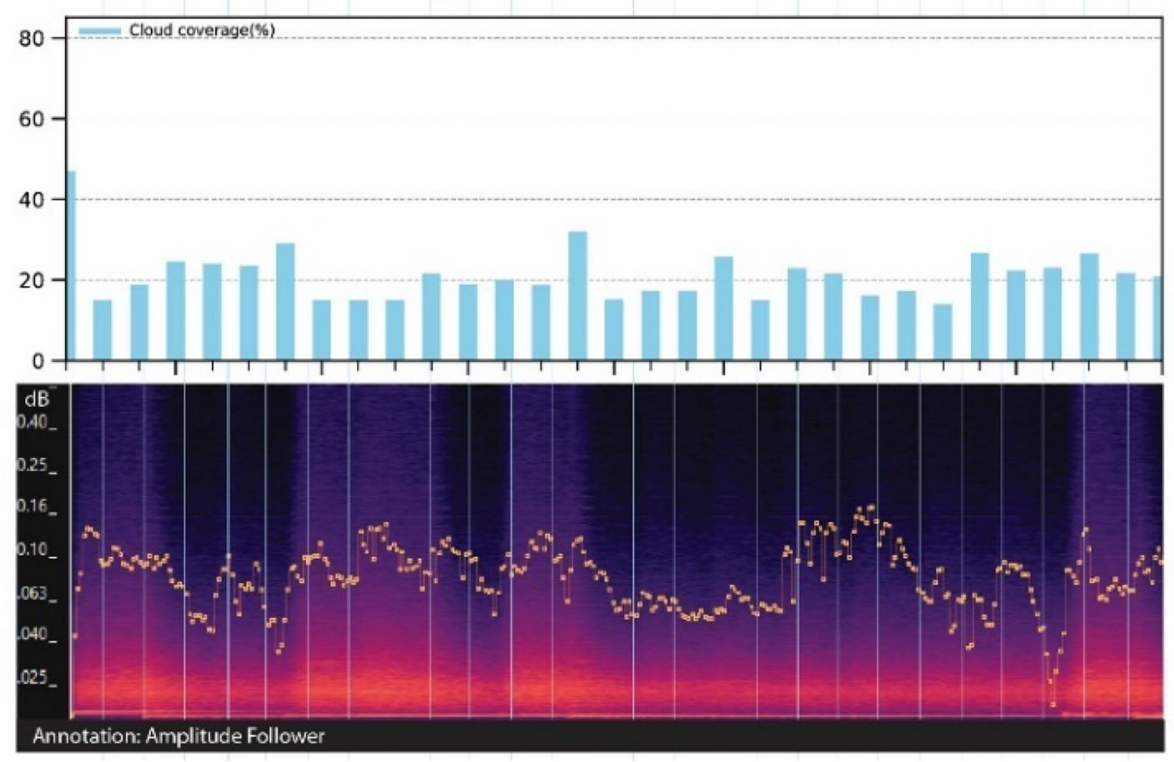

Figure 7. Comparison of changes in cloud coverage data and changes in amplitudes.

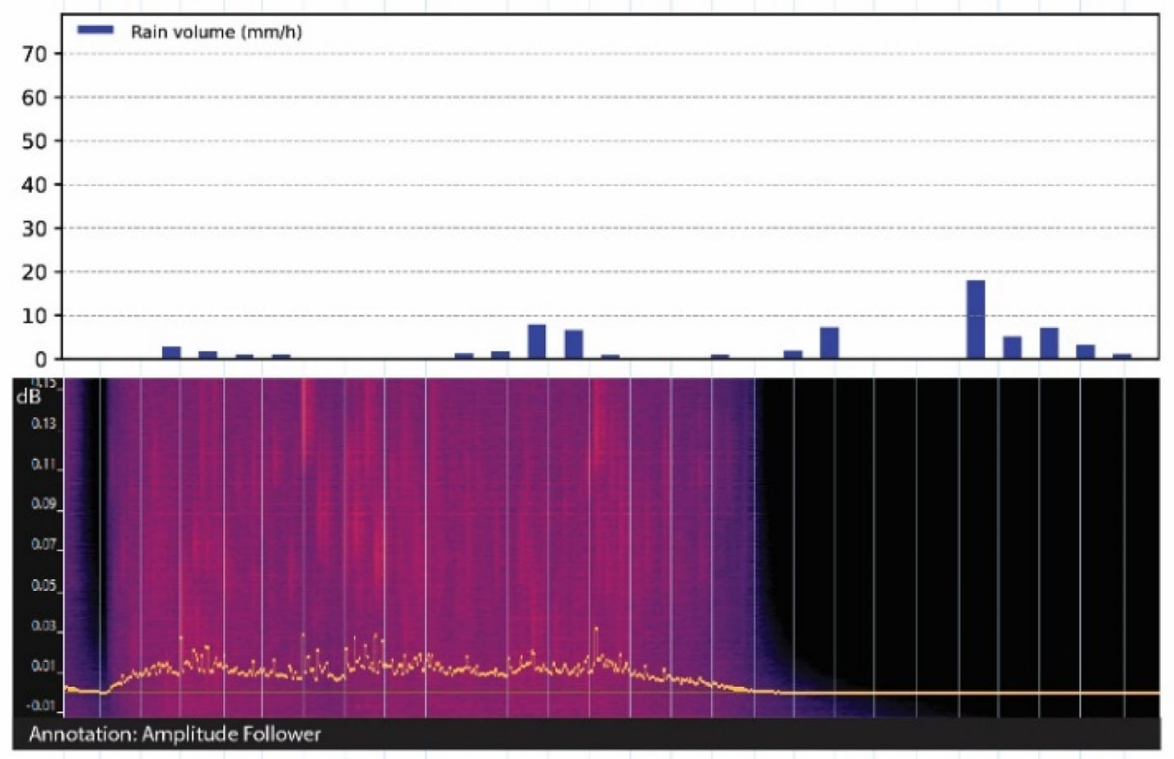

Figure 8. Comparison of changes in the volume of rain and changes in amplitudes.

parameters. However, the output altered if all processes were outputted at once, the combination of different sonic qualities will generate a new sonic event; abstracting the changes in data values, thus blurring the relation between the parameters.

Ultimately, both methods were found to be intricately linked to one another in the processes of creating sonic works. The transition from one method to another is found to be fluid and interchangeable as these methods consist of other undiscussed variables. These uncertainties can be further explored as creative avenues.

\section{Discussion/conclusions}

This paper suggested that environmental events can be perceived from a non-scientific perspective in creative practice. 
These methods enable knowledge, experience and sensibilities of the environment to be delivered through the creation of sonic works.

In the process of creation, the noise and weather events were highlighted as results of non-human geophysical influences. As such, noise was used as an aesthetic component to represent the irregularities of weather events. By tapping into the emancipatory aesthetic possibilities of noise, this paper offers an alternative way of environmental learning in contrast with the romantic environmentalist vision as prioritized in previous soundscape research.

In other respects, sonification was highlighted as a technical process that blurs the boundaries between scientific and artistic representation, in which relationships could be drawn in between. Sonification was a method proposed to realize the sensibilities of weather data; it also possessed the possibilities to generate new sensibilities and understanding each time when the process was executed. In contemporary sound art practice, sonification could be a useful method to reflect and apprehend the uncertainties of environmental event crises.

\section{Future works}

This paper only discusses limited scopes of non-human perspectives. With the flexibility of the proposed methods, we foresee the discussion and exploration to be stretched out to other types of quantifiable environmental data.

\section{Data availability}

Github: Underlying data for 'Sonification of weather data as non-human-centric artistic approach', https://github.com/ yikee95/weather-data-sonification

This project contains the following data:

- Pd-patch

- CSV-file.pd

- CSV-parse.pd

- Main.pd

- Save-audio-2.pd

Distribution License is GPL3

\section{Author contributions}

Ng Yi Kee: Conceptualization, Investigation, Methodology, Data Curation, Software, Visualization, Writing - Original Draft Preparation;

Lim KokYoong: Funding Acquisition, Supervision, Validation, Writing - Review and editing

\section{Acknowledgements}

We would like to thank Multimedia University for providing this opportunity and support for us to publish this study.

\section{References}

1. Nardi C: The Sound Studies Reader (ed. Jonathan Sterne). Dancecult. 2013; 5: 80-85.

Publisher Full Text

2. Schafer RM: The Soundscape: Our Sonic Environment and the tuning of the world. Destiny Books; 1993.

3. Oliveros P: Sonic Meditations. Smith Publications; 1974.

4. Novak D, Sakakeeny M: Keywords in sound. Duke University Press; 2015.
5. Latour B: Reassembling the Social: An Introduction to actor-networktheory. Oxford: OUP; 2007.

6. Oliveros P: Deep listening: A composer's sound practice. iUniverse; 2005.

7. OpenWeatherMap.org. (n.d.). Accuracy and quality of weather data. OpenWeatherMap. Retrieved January 13, 2022. Reference Source

8. Hermann T, Hunt A, Neuhoff JG: The sonification handbook. Logos Verlag Berlin; 2011. 


\section{Open Peer Review}

\section{Current Peer Review Status: $\mathrm{X}$}

\section{Version 1}

Reviewer Report 24 March 2022

https://doi.org/10.5256/f1000research.77199.r124361

(C) 2022 Oberman T. This is an open access peer review report distributed under the terms of the Creative Commons Attribution License, which permits unrestricted use, distribution, and reproduction in any medium, provided the original work is properly cited.

\section{Tin Oberman}

UCL Institute for Environmental Design and Engineering, The Bartlett, University College London, London, UK

This text features a description of an art project about sonification of weather data, written to fit the usual structure of a research article. With some modifications it could be a technical specification, but more modifications would be needed to become a research article. The technical specification could be a valuable addition to the body of knowledge on sonification.

While I found the artistic approach intriguing, while writing this review, I didn't try to assess its artistic value but simply look at its scientific soundness. I'm afraid I wasn't able to recognise a valid research question, nor a scientifically sound connection between the results presented and the conclusions, hence the above mentioned recommendation about the technical specification format.

The literature used is scarce. The introduction is very general and doesn't provide enough context for the work presented in the methods and results sections. The details provided are not sufficient to ensure reproducibility and there are mistakes in the analysis and data interpretation ( $\mathrm{dB}$ values in Figures $2 \& 3$ and Table 1). Steps laid out in the methods section are arbitrary, as is characteristic for an art work. Adding a reverberation effect to the part of the algorithm dedicated to sonification of clouds is a good example of this.

The purpose of mentioning the 'non-human-centric artistic approach' in the title is unclear.

The Abstract would need to be significantly improved.

Background doesn't seem to address the specific topic of the paper. The term 'sounding' seems a bit vague and it is unclear whether there are two or just one method as they feed into each other and the work presented doesn't appear to be valid without both. The abstract should be saying much more about what non-human-centric perspectives are and why they would be important. The role of sonification in this needs to be clearly described. Moreover, it seems that a lot of focus 
has been given to human perception throughout the work (choosing the timbre of the oscillators and deciding on how they will be modulated), one could even say that the sonification of weather data was clearly driven by human perception, so the non-human-centric part becomes even less clear.

The second paragraph of the introduction doesn't seem to take into account the body of soundscape research that very broadly evolved in the last 15-20 years around the interdisciplinary work relating to the ISO 12913 series. I have suggested some papers that could then lead into several soundscape-related directions ${ }^{1234}$.

The background described is very general and doesn't provide enough specifics related to the main work reported in this paper. I would suggest reshaping the introduction so it very clearly points out to the aims behind the main work reported. The key would be to describe other similar projects and reasons behind choosing this specific approach and methodology. Only the work by two composers is reported but activities such as listening and recording sounds and soundwalking go much beyond musical composition and this should be acknowledged. Further, as this seems to be the key, more space should be allowed to describe the non-human actants and how are they engaged in the soundscape framework.

The description of the Zoom H1n is vague and doesn't provide sufficient technical detail. Was the authors' intention to say that the recording doesn't accurately capture the dynamic range as experienced by the operators on-site? They should say so in much clearer words.

Moreover, the methods should be described much more clearly. Currently it seems that the recording took place before soundwalking which doesn't seem to be possible. Perhaps a diagram would be helpful.

Who perceived the surrounding ambience as noise and insignificant? The researchers? Was that a sampling strategy?

The details of where and when the soundwalks took place would be crucial to ensure replicability.

Perhaps there is no need to describe checking the environmental data online as an 'environmental engagement'. Monitoring and collecting weather data is a usual part of any soundwalk as per ISO/TS 12913-2.

How was the categorisation performed? Please elaborate in much higher detail.

A spectrogram combining amplitude and frequency would be much more useful in Figures 2 and 3.

The y scales in Figures 1 and 2, as well as the Table 1 look wrong, the dB values are impossibly small, the data should be checked.

Pure Data is indeed a open-source visual programming environment that can be used for sonification but more precision would be needed here to describe the specific patch that was used for the task in question. It doesn't seem like it is properly referenced. 
The coding applied in the Table 2 seems arbitrary, and while a certain musical and perceptual logic was given in the following paragraphs, it is not clear how those decisions serve the purpose of the paper. Further, white noise is a very harshly sounding waveform per se so it is not clear why it was used with a sine wave oscillator and why for instance low pass filtering was not considered in the design. Instead of adding reverberation, perhaps different attack and decay/release values could have been considered to enhance the perception of smoothness. Perhaps a complex delay processor could have been considered. This all only points out how arbitrary the process was. I would suggest to change the narrative to the point where all the artistic actions wouldn't be calling to be put under scrutiny but taken as they were, while the focus of the paper could become its potential implementation, perception or similar, if the authors do not decide to take the path of a technical specification/report.

The vague points in the results and conclusions - how exactly was noise highlighted as a prevalent sonic medium inspiring the creation of sound generators? What kind of a relation was drawn between environmental components and (which?) perceived intricacies? Was there any indication that environmental events couldn't be used in a sound-related practice? While weather might not be entirely predictable, it surely can be predicted in certain time series. For instance, if we can forecast daily or weekly weather condition with some certainty, why were the 5-7 minute recordings considered to be relevant to depict the irregularity of the weather? Keeping in mind the body of soundscape research available in the recent years, I am not certain why are the authors referring to it as a 'romantic environmentalist vision'.

Figure 4 is low quality and not all important parts are visible.

English language is excellent.

\section{References}

1. Zhao S, Qiu X, Lacey J, Maisch S: Configuring fixed-coefficient active control systems for traffic noise reduction. Building and Environment. 2019; 149: 415-427 Publisher Full Text

2. Ouzounian G, Lappin S: Soundspace: A Manifesto. Architecture and Culture. 2014; 2 (3): 305-316 Publisher Full Text

3. Aletta F, Kang J, Axelsson Ö: Soundscape descriptors and a conceptual framework for developing predictive soundscape models. Landscape and Urban Planning. 2016; 149: 65-74 Publisher Full Text

4. Jeon JY, Hong JY, Lee PJ: Soundwalk approach to identify urban soundscapes individually.J Acoust Soc Am. 2013; 134 (1): 803-12 PubMed Abstract | Publisher Full Text

Is the work clearly and accurately presented and does it cite the current literature? No

Is the study design appropriate and is the work technically sound? No

Are sufficient details of methods and analysis provided to allow replication by others? No

If applicable, is the statistical analysis and its interpretation appropriate? 


\section{Not applicable}

Are all the source data underlying the results available to ensure full reproducibility? No

Are the conclusions drawn adequately supported by the results?

No

Competing Interests: No competing interests were disclosed.

Reviewer Expertise: soundscape research, environmental acoustics, landscape architecture, spatial audio.

I confirm that I have read this submission and believe that I have an appropriate level of expertise to state that I do not consider it to be of an acceptable scientific standard, for reasons outlined above.

The benefits of publishing with F1000Research:

- Your article is published within days, with no editorial bias

- You can publish traditional articles, null/negative results, case reports, data notes and more

- The peer review process is transparent and collaborative

- Your article is indexed in PubMed after passing peer review

- Dedicated customer support at every stage

For pre-submission enquiries, contact research@f1000.com 